\title{
Unfit for work or alternate duties: what predicts the type of medical certificate for injured workers in Victoria, Australia
}

\author{
Rasa Ruseckaite \\ Institute for Safety, Compensation \& Recovery Research, Melbourne VIC 3004, Australia. rasa.ruseckaite@monash.edu
}

Background: General Practitioners (GPs) play an important role in worker's treatment and return-to-work (RTW).

Objectives: To establish what factors potentially predict the type of medical certification that GPs provide to injured workers following work-related injury.

Methods: A logistic regression analysis was performed to assess the impact of a number of factors on the likelihood that an injured worker would receive an alternate (ALT) vs. Unfit for work (UFW) duties certificate from their GP. Compensation Research Database, containing claims and medical certification data of Victorian injured workers was accessed for the research purposes.

Results: A total of 119,900 claims were included into this study. The majority of the injured workers were males, mostly age of 45-54 years. Nearly half of the workers with UFW and 36.9\% with ALT certificates had musculoskeletal injuries. The regression analysis revealed that older males were less likely to receive ALT as opposed to the younger females (25-34 years old) in most occupations. Living in rural areas was associated with smaller odds of receiving ALT. We also found that seeing a GP who is more experienced with workers' compensation increased the odds of ALT certificates. However, suffering from mental health issues decreased the odds of receiving the ALT in all workers.

Discussion: To our knowledge this is the first study to describe factors predicting GP medical certification of injured workers. The results clearly indicate that workers with physical injuries, female workers, and workers visiting GPs with a higher injured worker case load in metropolitan area are more likely to receive an ALT certificate.

Conclusions: The findings of this study help to identify groups of injured workers that are less likely to be recommended ALT certificates. It also suggests that certain health service providers and policy makers might require more education on the health benefits of RTW. 